
\title{
$\begin{array}{ll}\text { Research Square } & \begin{array}{l}\text { Preprints are preliminary reports that have not undergone peer review. } \\ \text { They should not be considered conclusive, used to inform clinical practice, } \\ \text { or referenced by the media as validated information. }\end{array}\end{array}$
}

\section{The direct and indirect impact of COVID-19 pandemic on maternal and child health services in Africa: a scoping review}

Prince Adu ( $\sim$ prince.adu@ubc.ca )

The University of British Columbia School of Population and Public Health https://orcid.org/0000-0002-3983-0875

Lisa Stallwood

The University of British Columbia School of Population and Public Health

Stephen O. Adebola

Ladoke Akintola University of Technology Teaching Hospital: LAUTECH Teaching Hospital

Theresa Abah

California State University Sacramento

Amold Ikedichi Okpani

The University of British Columbia School of Population and Public Health

\section{Research Article}

Keywords: COVID-19, Africa, maternal and child health, reproductive health

Posted Date: August 10th, 2021

DOI: https://doi.org/10.21203/rs.3.rs-597730/v1

License: (c) (7) This work is licensed under a Creative Commons Attribution 4.0 International License. Read Full License 


\section{Abstract}

\section{Introduction}

The novel coronavirus disease 2019 (COVID-19) continues to disrupt the availability and utilization of routine and emergency health care services, with differing impacts in jurisdictions across the world. In this scoping review, we set out to synthesize documentation of the direct and indirect effect of the pandemic, and national responses to it, on maternal and child health $(\mathrm{MNCH})$ in Africa.

\section{Methods}

A scoping review was conducted to provide an overview of the most significant impacts identified up to February 2021. Our search included peer reviewed literature, working papers and news articles with the intent to capture evolving and novel updates on the topic. Narrative descriptions were written about thematic areas for which the authors extracted the most evidence.

\section{Results}

One hundred and fifty-five articles were identified through our literature search and 17 were deemed appropriate for analysis. We identified three overarching themes: delayed care, disruption in service provision and utilization and mitigation strategies or recommendation.

\section{Discussion}

Available literature suggests widespread service cutbacks amid a mosaic of mitigation strategies. Our results show that minor consideration was given to preserving health service access for mothers and children, especially in historically underserved areas. Lack of emergency preparedness and infrastructural constraints prevented useful approaches such as telemedicine from being meaningfully utilized.

\section{Conclusions}

Our scoping review shows that limited research has been published on this topic. Reviewed literature illuminates the continuing need and prioritization for maternity services, immunization, and reproductive health services, which have not been prioritized during the pandemic, yet are necessary to shield the continent's most vulnerable population segments from the shocks of current and future global health emergencies.

\section{Introduction}

First reported in the final weeks of 2019, the novel severe acute respiratory syndrome coronavirus 2 (SARS-CoV-2) continued to spread the globe. On March 11, 2020, the World Health Organization (WHO) declared the outbreak a global pandemic (1). The declaration by the WHO was quickly followed in jurisdictions across the world, by the implementation of national control measures $(2,3)$. Whilst some of the control measures necessitated partial or complete shutdown of economic activities, arguably some of the most difficult decisions to navigate at the start of the pandemic were regarding health care services (4). Decisionmakers had to quickly determine how to keep health facilities running while protecting health care workers, our heroes whom COVID-19 patients depended on for the reception of hospital care.

In many instances, health care facilities were closed to 'routine' services as part of pandemic mitigation measures (5). For African populations, this is a troubling disruption to an already overburdened sector. African countries had witnessed the devastating impact of communicable disease outbreaks on health service provision and health outcomes from the human immunodeficiency virus (HIV) and tuberculosis in South Africa $(6,7)$, to Ebola virus disease in several West African states (8). Expectedly, the consequences of the health service disruptions have been disproportionately borne by the most vulnerable population groups: women, mothers, and children.

Prior to the start of the COVID-19 pandemic, health systems in Africa have been strained from the burden of historic underinvestment $(9,10)$. The attendant weaknesses meant that countries were not on track to achieve global human health development goals - including the health-related Sustainable Development Goals (11). Though some progress had been made over the course of the last few decades, the region continued to have some of the poorest maternal, newborn, and child health outcomes globally $(12,13)$.

The COVID-19 pandemic has impacted maternal, newborn and child health (MNCH) care service availability and uptake in Africa. There are reports of widespread avoidance of health care facilities for fear of being diagnosed with COVID-19 or contracting it from health care workers or other patients (14, 15). In regions where services have not been outrightly closed, many health facilities are operating at very limited capacity $(15,16)$. HCWs have carried the burden of COVID-19 infections, leading to absence from work, and in some tragic cases, many have died from the disease. Furthermore, human and health care resources have been diverted away from $\mathrm{MNCH}$ to support emergency response activities (17).

Events beyond the health sector are expected to have a knock-on effect on $\mathrm{MNCH}$ outcomes. At the macro level, with the economic contraction triggered by the pandemic, it is reasonable to expect that health sector investments in the short and long terms will be further constrained especially in low-and middle-income countries. The net impact of the pandemic is projected to not only halt progress on improving MNCH outcomes in Africa, but to potentially reverse decades of gains (18). Added to the foregoing are concerns of the child developmental impact of lockdowns and school closures $(17,19)$.

It is expected that recovery from the impact of the pandemic will be more drawn out in Africa. Nations are counting on vaccinating enough of their populations to reach herd immunity - an important pathway for safe reopening and economic recovery. Vaccine roll-out trends, however, indicate that African countries 
will substantially lag others in terms of widespread availability and distribution of vaccines $(20,21)$, resulting in prolonged health service disruption and further constraints on $\mathrm{MNCH}$, relative to high income countries.

\section{Rationale for conducting this review}

In responding to the pandemic, national governments, non-governmental and private sector actors have adopted varying strategies regarding health care service provision. Adaptations have ranged from the transition to remote/telehealth services to continuing normal operations. Now that the pandemic is in its second year, sufficient time has passed, and knowledge has evolved substantially to the point that lessons can be learned and shared on what has worked, or not, for keeping health services running in resource constrained settings.

The impact of the COVID-19 pandemic on the health of women, mothers and children is a subject of several articles and commentaries (15, 22, 23). To our knowledge, there has not been a scoping review effort to date to collect documentation of the effect of the COVID-19 pandemic on the availability and demand for $\mathrm{MNCH}$ services in Africa, whilst simultaneously highlighting the strategies that have been adopted by actors on the continent to sustain service provision and uptake.

\section{Objectives}

This paper synthesizes available documentation on the impacts of the COVID-19 pandemic on MNCH service availability, utilization, and outcomes in Africa. We aim to document the proposed strategies in different African countries for continuing MNCH services during the COVID-19 pandemic. We hope that these lessons can be applied to help increase awareness of how the health and wellbeing of mothers and children in Africa have been impacted due to the pandemic.

\section{Methods}

This scoping review followed the framework proposed by Arksey and O'Malley (24) to map all the evolving literature on our research topic. We followed the following five stages of review: identify research question, identify relevant studies, select studies to review, chart the data and collate, summarize, and report results.

\section{Identify research question}

Through a preliminary search and consultation with our team members, the following research question was developed: How has the COVID-19 pandemic directly and indirectly impacted maternal and child health in Africa?

\section{Identify relevant studies}

A comprehensive search strategy was developed to identify relevant papers from the following electronic databases: Ovid: MEDLINE, Ovid: Embase, and Ovid: HealthSTAR. Literature that was published in English from January 1, 2020, to February 10, 2021, was included in the review process. Google scholar was also used with its own search strategy to capture grey literature, working papers and any other papers which could have been missed by the other databases. Our Google Scholar search captured all relevant literature published in English from January 1, 2020 to February 27, 2021. Bibliographies were scanned to capture any studies that were missed with our formal search (See Additional file 1 for detailed search strategy).

\section{Study selection}

All articles that our search strategy returned which were on the direct or indirect impacts of COVID - 19 on maternal and child health in Africa, written in English, and published within our established dates were included. For any identified duplicates, we included the copy with a more complete description. The screening process was executed in two stages. Firstly, the title and abstract of each article was independently screened by two authors and each study was marked as 'yes', 'no' or 'unclear'. A study was marked as unclear if its relevance to our topic was unclear upon title and abstract screen. All studies marked as 'yes' or 'unclear' were included for full-text review. All available full-text articles were independently reviewed by two authors. Any article that mentioned maternal and child health or a synonym that was used in our search strategy, mentioned COVID-19, and concentrated on the African continent was included. Studies that were not focused on Africa but only mentioned an African country or setting were excluded. Covidence systematic review software was used for deduplication, screening, and data extraction.

\section{Chart the Data}

Seventeen (17) articles were included in the review. These articles included commentaries, review articles, retrospective studies, cross sectional studies, mixedmethod studies, an editorial letter, and clinical guidelines. Preliminary themes were developed after an initial read of the full text articles and themes were discussed amongst all authors. Two authors coded each article independently and discussed the codes with the team prior to charting them under the appropriate theme. Standard information about each article including title, topic, setting and outcome of interest were recorded in a Microsoft Excel file.

\section{Collate, summarize, and report results}

The main findings and recommendations by the authors of the included studies were tabulated and narrative descriptions of each article were written. All authors reviewed the themes with corresponding narratives to improve readability.

\section{Results}

\section{Search Results}


Applying the appropriate search filters, a total of 209 articles were identified from our selected databases. Of these, 51 articles and reports were eligible for fulltext screening. After the full text review, 34 were excluded because they were either not focused on health service utilization (twelve), either only mentioned $\mathrm{MNCH}$ without an entire focus (twelve), did not focus on Africa (eight) or only proposed a research protocol on this topic (two). The remaining 17 were included in this scoping review. The selection process is exemplified in the PRISMA flow diagram (Fig. 1).

\section{Characteristics of included articles/studies}

The article type varied vastly as shown in Table 1 . Of these, commentaries were the most common $(n=5 ; 29 \%)$; majority of articles were published in peer reviewed journals $(n=13,76 \%)$. 
Table 1

Studies included in the scoping review

\begin{tabular}{|c|c|c|c|c|c|c|c|c|}
\hline Author/ Date & Title & Joumal & $\begin{array}{l}\text { Type of } \\
\text { study/report }\end{array}$ & $\begin{array}{l}\text { Peer } \\
\text { review } \\
\text { status }\end{array}$ & Topic & Setting & $\begin{array}{l}\text { Outcome of } \\
\text { interest }\end{array}$ & Main message \\
\hline $\begin{array}{l}\text { Jensen et al., } \\
\text { February } 2021\end{array}$ & $\begin{array}{l}\text { Child health } \\
\text { services } \\
\text { during a } \\
\text { COVID-19 } \\
\text { outbreak in } \\
\text { KwaZulu- } \\
\text { Natal } \\
\text { Province, } \\
\text { South Africa }\end{array}$ & $\begin{array}{l}\text { South } \\
\text { African } \\
\text { Medical } \\
\text { Journal }\end{array}$ & $\begin{array}{l}\text { Retrospective } \\
\text { study }\end{array}$ & $\begin{array}{l}\text { Peer } \\
\text { reviewed }\end{array}$ & $\begin{array}{l}\text { Direct and } \\
\text { Indirect } \\
\text { effects of } \\
\text { COVID-19 on } \\
\text { children }\end{array}$ & South Africa & $\begin{array}{l}\text { Child health } \\
\text { services }\end{array}$ & $\begin{array}{l}\text { COVID-19 has led } \\
\text { a disruption in } \\
\text { service access an } \\
\text { utilization, service } \\
\text { delivery and child } \\
\text { well-being. There } \\
\text { has also been } \\
\text { reduction in hospi } \\
\text { admissions. }\end{array}$ \\
\hline $\begin{array}{l}\text { Gray et al., } \\
\text { January } 2021\end{array}$ & $\begin{array}{l}\text { COVID-19 } \\
\text { and Pediatric } \\
\text { Lung } \\
\text { Disease: A } \\
\text { South African } \\
\text { Tertiary } \\
\text { Center } \\
\text { Experience }\end{array}$ & $\begin{array}{l}\text { Frontiers in } \\
\text { pediatrics }\end{array}$ & $\begin{array}{l}\text { Literature } \\
\text { review }\end{array}$ & $\begin{array}{l}\text { Peer } \\
\text { reviewed }\end{array}$ & $\begin{array}{l}\text { Direct and } \\
\text { Indirect } \\
\text { effects on } \\
\text { under-18-year } \\
\text { old }\end{array}$ & South Africa & $\begin{array}{l}\text { Child health } \\
\text { outcomes with } \\
\text { chronic lung } \\
\text { disease globally }\end{array}$ & $\begin{array}{l}\text { Pre-existing } \\
\text { childhood } \\
\text { respiratory diseas } \\
\text { does not appear tı } \\
\text { be significantly } \\
\text { associated with } \\
\text { severe COVID-19 } \\
\text { disease. } \\
\text { Longitudinal data } \\
\text { needed to assess } \\
\text { the risk of COVID1 } \\
19 \text { in children witt } \\
\text { immunosuppressi } \\
\text { and interstitial lun } \\
\text { diseases. }\end{array}$ \\
\hline $\begin{array}{l}\text { Oluwasola et } \\
\text { al., January } \\
2021\end{array}$ & $\begin{array}{l}\text { COVID-19 } \\
\text { and its } \\
\text { implications } \\
\text { for obstetrics } \\
\text { and } \\
\text { gynecology } \\
\text { practice in } \\
\text { Africa }\end{array}$ & $\begin{array}{l}\text { Pan African } \\
\text { Medical } \\
\text { Journal }\end{array}$ & A review & $\begin{array}{l}\text { Peer } \\
\text { reviewed }\end{array}$ & $\begin{array}{l}\text { Direct and } \\
\text { Indirect } \\
\text { effects on } \\
\text { pregnancy, } \\
\text { labour and } \\
\text { delivery }\end{array}$ & Africa & $\begin{array}{l}\text { Obstetrics and } \\
\text { gynecologic } \\
\text { practice in Africa }\end{array}$ & $\begin{array}{l}\text { The COVID-19 } \\
\text { pandemic will } \\
\text { negatively affect } \\
\text { obstetrics and } \\
\text { gynecology } \\
\text { practices. Some o } \\
\text { the effects includt } \\
\text { reduced antenatal } \\
\text { attendance and } \\
\text { disruption in routi } \\
\text { essential services } \\
\text { The provision and } \\
\text { utilization of } \\
\text { reproductive, } \\
\text { maternal, newborr } \\
\text { and child health } \\
\text { services are likely } \\
\text { be reduced. }\end{array}$ \\
\hline $\begin{array}{l}\text { Kotlar et al., } \\
\text { January } 2021\end{array}$ & $\begin{array}{l}\text { The impact } \\
\text { of the COVID- } \\
19 \text { pandemic } \\
\text { on maternal } \\
\text { and perinatal } \\
\text { health: a } \\
\text { scoping } \\
\text { review }\end{array}$ & $\begin{array}{l}\text { Reproductive } \\
\text { health }\end{array}$ & $\begin{array}{l}\text { Scoping } \\
\text { review }\end{array}$ & $\begin{array}{l}\text { Peer } \\
\text { reviewed }\end{array}$ & $\begin{array}{l}\text { Direct and } \\
\text { Indirect } \\
\text { effects of on } \\
\text { maternal and } \\
\text { perinatal } \\
\text { health }\end{array}$ & Worldwide & $\begin{array}{l}\text { Maternal and } \\
\text { perinatal health }\end{array}$ & $\begin{array}{l}\text { Apart from the dir } \\
\text { effects of COVID-- } \\
\text { on reproductive ar } \\
\text { perinatal health, } \\
\text { indirect impacts } \\
\text { resulting from } \\
\text { changes in health } \\
\text { care, social, and } \\
\text { economic situatio } \\
\text { have been noted. } \\
\text { The health } \\
\text { outcomes of } \\
\text { pregnant women } \\
\text { who have } \\
\text { symptomatic } \\
\text { CoviD-19 may be } \\
\text { more severe than } \\
\text { people who are nc } \\
\text { pregnant. }\end{array}$ \\
\hline $\begin{array}{l}\text { Tadesse et al., } \\
\text { December } \\
2020\end{array}$ & $\begin{array}{l}\text { Antenatal } \\
\text { Care Service } \\
\text { Utilization of } \\
\text { Pregnant } \\
\text { Women } \\
\text { Attending } \\
\text { Antenatal } \\
\text { Care in Public } \\
\text { Hospitals } \\
\text { During the } \\
\text { COVID-19 } \\
\text { Pandemic } \\
\text { Period }\end{array}$ & $\begin{array}{l}\text { International } \\
\text { journal of } \\
\text { women's } \\
\text { health }\end{array}$ & $\begin{array}{l}\text { Cross- } \\
\text { sectional } \\
\text { study }\end{array}$ & $\begin{array}{l}\text { Peer } \\
\text { reviewed }\end{array}$ & $\begin{array}{l}\text { Direct and } \\
\text { Indirect } \\
\text { effects on } \\
\text { pregnancy, } \\
\text { labour and } \\
\text { delivery }\end{array}$ & Ethiopia & $\begin{array}{l}\text { Antenatal care } \\
\text { utilization }\end{array}$ & $\begin{array}{l}\text { Antenatal care } \\
\text { utilization was } \\
\text { negatively impact } \\
\text { by maternal age; } \\
\text { residency status; } \\
\text { educational statu: } \\
\text { still birth history; } \\
\text { maternity service } \\
\text { diversion and } \\
\text { interruption, COVII } \\
19 \text { pandemic fear, } \\
\text { and transportatiol } \\
\text { challenges. }\end{array}$ \\
\hline
\end{tabular}




\begin{tabular}{|c|c|c|c|c|c|c|c|c|}
\hline Author/ Date & Title & Joumal & $\begin{array}{l}\text { Type of } \\
\text { study/report }\end{array}$ & $\begin{array}{l}\text { Peer } \\
\text { review } \\
\text { status }\end{array}$ & Topic & Setting & $\begin{array}{l}\text { Outcome of } \\
\text { interest }\end{array}$ & Main message \\
\hline $\begin{array}{l}\text { Murewanhema } \\
\text { et al., } \\
\text { November } \\
2020\end{array}$ & $\begin{array}{l}\text { Restoring and } \\
\text { maintaining } \\
\text { robust } \\
\text { maternity } \\
\text { services in } \\
\text { the COVID-19 } \\
\text { era: a public } \\
\text { health } \\
\text { dilemma in } \\
\text { Zimbabwe }\end{array}$ & $\begin{array}{l}\text { Pan African } \\
\text { Medical } \\
\text { Journal }\end{array}$ & Commentary & $\begin{array}{l}\text { Peer } \\
\text { reviewed }\end{array}$ & $\begin{array}{l}\text { Direct effects } \\
\text { on pregnancy, } \\
\text { labour and } \\
\text { delivery }\end{array}$ & Zimbabwe & $\begin{array}{l}\text { Maternity service } \\
\text { utilization and } \\
\text { recommendations }\end{array}$ & $\begin{array}{l}\text { Restricting access } \\
\text { to maternity care } \\
\text { may have more di } \\
\text { consequences tha } \\
\text { COVID-19. There i: } \\
\text { an urgent need to } \\
\text { restore and } \\
\text { maintain full } \\
\text { maternity services } \\
\text { in line with the } \\
\text { World Health } \\
\text { Organization's } \\
\text { guidance on } \\
\text { maintaining } \\
\text { essential health } \\
\text { services during th } \\
\text { COVID-19 era. }\end{array}$ \\
\hline $\begin{array}{l}\text { Pires et al., } \\
\text { November } \\
2020\end{array}$ & $\begin{array}{l}\text { COVID-19 } \\
\text { Pandemic } \\
\text { Impact on } \\
\text { Maternal and } \\
\text { Child Health } \\
\text { Services } \\
\text { Access in } \\
\text { Nampula, } \\
\text { Mozambique: } \\
\text { A Mixed } \\
\text { Methods } \\
\text { Research. }\end{array}$ & $\begin{array}{l}\text { Research } \\
\text { Square } \\
\text { preprint }\end{array}$ & $\begin{array}{l}\text { Mixed } \\
\text { methods } \\
\text { research }\end{array}$ & Preprint & $\begin{array}{l}\text { Direct and } \\
\text { Indirect } \\
\text { effects of pre- } \\
\text { pregnancy, } \\
\text { pregnancy, } \\
\text { labor and } \\
\text { delivery }\end{array}$ & Mozambique & $\begin{array}{l}\text { Access to } \\
\text { maternal and } \\
\text { children health } \\
\text { services }\end{array}$ & $\begin{array}{l}\text { COVID-19 has } \\
\text { negatively affecte } \\
\text { maternal and chilı } \\
\text { health; reductions } \\
\text { maternity hospita } \\
\text { deliveries, childho } \\
\text { vaccination, famil } \\
\text { planning visit, ant } \\
\text { natal visit and } \\
\text { increases in hom€ } \\
\text { deliveries. }\end{array}$ \\
\hline $\begin{array}{l}\text { Banke- } \\
\text { Thomas et al., } \\
\text { November } \\
2020\end{array}$ & $\begin{array}{l}\text { Utilization } \\
\text { cost of } \\
\text { maternity } \\
\text { services for } \\
\text { childbirth } \\
\text { among } \\
\text { pregnant } \\
\text { women with } \\
\text { coronavirus } \\
\text { disease } 2019 \\
\text { in Nigeria's } \\
\text { epicenter }\end{array}$ & Obstetrics & $\begin{array}{l}\text { Cross- } \\
\text { sectional } \\
\text { study } \\
\text { (Hospital- } \\
\text { based cost } \\
\text { analysis) }\end{array}$ & $\begin{array}{l}\text { Peer } \\
\text { reviewed }\end{array}$ & $\begin{array}{l}\text { Indirect } \\
\text { effects on } \\
\text { pregnancy }\end{array}$ & Nigeria & $\begin{array}{l}\text { Maternal services } \\
\text { utilization costs }\end{array}$ & $\begin{array}{l}\text { COVID-19 has } \\
\text { directly impacted } \\
\text { cost of maternity } \\
\text { service utilization } \\
\text { especially as } \\
\text { governmental } \\
\text { exemptions becor } \\
\text { unavailable, } \\
\text { reduction of } \\
\text { donations and } \\
\text { presence of fees } \\
\text { attributable to } \\
\text { universal testing. } \\
\text { Such huge costs c } \\
\text { care may become } \\
\text { unaffordable } \\
\text { especially for } \\
\text { pregnant women } \\
\text { with COVID-19. }\end{array}$ \\
\hline $\begin{array}{l}\text { Ogunkola et } \\
\text { al., November } \\
2020\end{array}$ & $\begin{array}{l}\text { Impact of } \\
\text { COVID-19 } \\
\text { pandemic on } \\
\text { antenatal } \\
\text { healthcare } \\
\text { services in } \\
\text { Sub-Saharan } \\
\text { Africa }\end{array}$ & $\begin{array}{l}\text { Public } \\
\text { Health in } \\
\text { Practice }\end{array}$ & $\begin{array}{l}\text { Letter to the } \\
\text { editor }\end{array}$ & $\begin{array}{l}\text { Not } \\
\text { applicable }\end{array}$ & $\begin{array}{l}\text { Direct and } \\
\text { Indirect } \\
\text { effects on } \\
\text { pregnancy }\end{array}$ & Africa & $\begin{array}{l}\text { Antenatal } \\
\text { healthcare } \\
\text { services }\end{array}$ & $\begin{array}{l}\text { African } \\
\text { governments mus } \\
\text { ensure antenatal } \\
\text { care services and } \\
\text { other related } \\
\text { healthcare service } \\
\text { are not disrupted } \\
\text { due to the urgent } \\
\text { need to contain th } \\
\text { COVID-19 } \\
\text { pandemic. }\end{array}$ \\
\hline $\begin{array}{l}\text { Pallangyo et } \\
\text { al., October } \\
2020\end{array}$ & $\begin{array}{l}\text { The impact } \\
\text { of COVID-19 } \\
\text { on midwives' } \\
\text { practice in } \\
\text { Kenya, } \\
\text { Uganda and } \\
\text { Tanzania: A } \\
\text { reflective } \\
\text { account }\end{array}$ & Midwifery & Commentary & $\begin{array}{l}\text { Peer } \\
\text { reviewed }\end{array}$ & $\begin{array}{l}\text { Direct and } \\
\text { Indirect } \\
\text { effects on } \\
\text { pregnancy, } \\
\text { labour and } \\
\text { delivery }\end{array}$ & $\begin{array}{l}\text { Kenya, } \\
\text { Tanzania, } \\
\text { Uganda }\end{array}$ & $\begin{array}{l}\text { Midwifery } \\
\text { practice }\end{array}$ & $\begin{array}{l}\text { COVID-19 pander } \\
\text { has exacerbated a } \\
\text { already elevated } \\
\text { maternal and } \\
\text { neonatal mortality } \\
\text { in East Africa. Thi } \\
\text { trend is likely to } \\
\text { continue in future } \\
\text { despite the best } \\
\text { efforts of midwiv€ } \\
\text { and other health } \\
\text { professionals with } \\
\text { likely resultant } \\
\text { upward surge in th } \\
\text { numbers of COVI[ } \\
19 \text { related deaths } \\
\text { reproductive age } \\
\text { women. }\end{array}$ \\
\hline
\end{tabular}




\begin{tabular}{|c|c|c|c|c|c|c|c|c|}
\hline Author/ Date & Title & Joumal & $\begin{array}{l}\text { Type of } \\
\text { study/report }\end{array}$ & $\begin{array}{l}\text { Peer } \\
\text { review } \\
\text { status }\end{array}$ & Topic & Setting & $\begin{array}{l}\text { Outcome of } \\
\text { interest }\end{array}$ & Main message \\
\hline $\begin{array}{l}\text { Wangamati et } \\
\text { al., September } \\
2020\end{array}$ & $\begin{array}{l}\text { The } \\
\text { ramifications } \\
\text { of COVID-19 } \\
\text { on maternal } \\
\text { health in } \\
\text { Kenya }\end{array}$ & $\begin{array}{l}\text { Sexual and } \\
\text { Reproductive } \\
\text { Health } \\
\text { Matters }\end{array}$ & Commentary & $\begin{array}{l}\text { Peer } \\
\text { reviewed }\end{array}$ & $\begin{array}{l}\text { Direct and } \\
\text { Indirect } \\
\text { effects on } \\
\text { pregnancy, } \\
\text { labour and } \\
\text { delivery }\end{array}$ & Kenya & $\begin{array}{l}\text { Maternal health } \\
\text { ramifications and } \\
\text { recommendations }\end{array}$ & $\begin{array}{l}\text { Resources meant } \\
\text { for maternal healt } \\
\text { services have bee } \\
\text { reallocation to cat } \\
\text { for COVID-19 } \\
\text { patients thereby } \\
\text { negatively affectir } \\
\text { maternal health } \\
\text { service delivery. T } \\
\text { absence of an } \\
\text { emergency } \\
\text { preparedness plar } \\
\text { due to inadequate } \\
\text { human and } \\
\text { financial resource } \\
\text { was underscored. }\end{array}$ \\
\hline $\begin{array}{l}\text { Shikuku et al., } \\
\text { September } \\
2020\end{array}$ & $\begin{array}{l}\text { Early indirect } \\
\text { impact of } \\
\text { COVID-19 } \\
\text { pandemic on } \\
\text { utilization } \\
\text { and } \\
\text { outcomes of } \\
\text { reproductive, } \\
\text { maternal, } \\
\text { newborn, } \\
\text { child and } \\
\text { adolescent } \\
\text { health } \\
\text { services in } \\
\text { Kenya }\end{array}$ & $\begin{array}{l}\text { MedRxiv } \\
\text { preprint }\end{array}$ & $\begin{array}{l}\text { Cross } \\
\text { sectional } \\
\text { study }\end{array}$ & Preprint & $\begin{array}{l}\text { Indirect } \\
\text { effects of } \\
\text { COVID-19 on } \\
\text { utilization } \\
\text { and } \\
\text { outcomes of } \\
\text { reproductive, } \\
\text { maternal, } \\
\text { newborn, } \\
\text { child and } \\
\text { adolescent } \\
\text { health } \\
\text { services }\end{array}$ & Kenya & $\begin{array}{l}\text { Hospital } \\
\text { attendance for } \\
\text { select } \\
\text { reproductive, } \\
\text { maternal, } \\
\text { newborn, child } \\
\text { and adolescent } \\
\text { health services }\end{array}$ & $\begin{array}{l}\text { The increase in } \\
\text { adolescent } \\
\text { pregnancy, } \\
\text { adolescent materr } \\
\text { death and still birt } \\
\text { rates in Kenya ma } \\
\text { be attributed to th } \\
\text { COVID-19 } \\
\text { pandemic. There } \\
\text { concerns about th } \\
\text { loss of recent gair } \\
\text { achieved in } \\
\text { maternal and } \\
\text { perinatal health. }\end{array}$ \\
\hline $\begin{array}{l}\text { Saso et al., } \\
\text { September } \\
2020\end{array}$ & $\begin{array}{l}\text { Impact of } \\
\text { COVID-19 on } \\
\text { Immunization } \\
\text { Services for } \\
\text { Maternal and } \\
\text { Infant } \\
\text { Vaccines: } \\
\text { Results of a } \\
\text { Survey } \\
\text { Conducted by } \\
\text { immunizing } \\
\text { Pregnant } \\
\text { Women and } \\
\text { Infants } \\
\text { Network }\end{array}$ & Vaccines & $\begin{array}{l}\text { Cross } \\
\text { sectional } \\
\text { study }\end{array}$ & $\begin{array}{l}\text { Peer } \\
\text { reviewed }\end{array}$ & $\begin{array}{l}\text { Indirect } \\
\text { effects on } \\
\text { immunization } \\
\text { services }\end{array}$ & Worldwide & $\begin{array}{l}\text { Maternal, child } \\
\text { and perinatal } \\
\text { immunization } \\
\text { services. }\end{array}$ & $\begin{array}{l}\text { Three key themes } \\
\text { underscore } \\
\text { immunization } \\
\text { disruptions as a } \\
\text { result of the COVII } \\
19 \text { pandemic: } \\
\text { access issues, } \\
\text { provider issues an } \\
\text { user concern or } \\
\text { COVID-19 fears. }\end{array}$ \\
\hline
\end{tabular}




\begin{tabular}{|c|c|c|c|c|c|c|c|c|}
\hline Author/ Date & Title & Joumal & $\begin{array}{l}\text { Type of } \\
\text { study/report }\end{array}$ & $\begin{array}{l}\text { Peer } \\
\text { review } \\
\text { status }\end{array}$ & Topic & Setting & $\begin{array}{l}\text { Outcome of } \\
\text { interest }\end{array}$ & Main message \\
\hline $\begin{array}{l}\text { Eghtessadi et } \\
\text { al., August } \\
2020\end{array}$ & $\begin{array}{l}\text { Safeguarding } \\
\text { gains in the } \\
\text { sexual and } \\
\text { reproductive } \\
\text { health and } \\
\text { AIDS } \\
\text { response } \\
\text { amidst } \\
\text { COVID-19: } \\
\text { The role of } \\
\text { African civil } \\
\text { society }\end{array}$ & $\begin{array}{l}\text { International } \\
\text { journal of } \\
\text { infectious } \\
\text { diseases }\end{array}$ & Commentary & $\begin{array}{l}\text { Peer } \\
\text { reviewed }\end{array}$ & $\begin{array}{l}\text { Indirect } \\
\text { effects on } \\
\text { pregnancy } \\
\text { and } \\
\text { adolescent } \\
\text { population }\end{array}$ & Africa & $\begin{array}{l}\text { Sexual and } \\
\text { reproductive } \\
\text { health }\end{array}$ & $\begin{array}{l}\text { Civil society } \\
\text { organizations mu: } \\
\text { be engaged in } \\
\text { efforts to mitigate } \\
\text { COVID-19 } \\
\text { detrimental effect } \\
\text { on sexual and } \\
\text { reproductive healt } \\
\text { (SRH) outcomes. } \\
\text { They should apply } \\
\text { the following five } \\
\text { principal actions: } \\
\text { accountability of } \\
\text { governments } \\
\text { around optimal us } \\
\text { of COVID-19 fund: } \\
\text { and resources; } 2 \text { ) } \\
\text { keeping sexual an } \\
\text { reproductive healt } \\
\text { and HIV central to } \\
\text { the Universal Heal } \\
\text { Coverage agenda; } \\
\text { lobbying } \\
\text { governments to } \\
\text { invest in innovativ } \\
\text { and good practice } \\
\text { models; } 4 \text { ) mitigat } \\
\text { the gendered imp; } \\
\text { of COVID-19 and! } \\
\text { advocate that } \\
\text { African } \\
\text { governments remi } \\
\text { vigilant in securin } \\
\text { access to new } \\
\text { therapeutics at } \\
\text { reasonable cost } \\
\text { from the global } \\
\text { market. }\end{array}$ \\
\hline $\begin{array}{l}\text { Nandi et al., } \\
\text { August } 2020\end{array}$ & $\begin{array}{l}\text { SARS-CoV-2 } \\
\text { in Malawi: } \\
\text { Are we } \\
\text { sacrificing } \\
\text { the Youth in } \\
\text { sub-Saharan } \\
\text { Africa? }\end{array}$ & $\begin{array}{l}\text { Journal of } \\
\text { global health }\end{array}$ & Commentary & $\begin{array}{l}\text { Peer } \\
\text { reviewed }\end{array}$ & $\begin{array}{l}\text { Indirect } \\
\text { effects on } \\
\text { childhood } \\
\text { population }\end{array}$ & Malawi & $\begin{array}{l}\text { Pediatric } \\
\text { admissions, } \\
\text { human resources } \\
\text { for health }\end{array}$ & $\begin{array}{l}\text { Public health } \\
\text { measures that ha' } \\
\text { been implementec } \\
\text { to prevent and } \\
\text { control the spread } \\
\text { of COVID-19 } \\
\text { disproportionally } \\
\text { affect the youth. } \\
\text { These measures } \\
\text { have resulted in tr } \\
\text { loss of national al } \\
\text { international } \\
\text { medical staff at o } \\
\text { of Africa's biggest } \\
\text { children's } \\
\text { department, } \\
\text { decrease in pediat } \\
\text { bed occupancy et } \\
\text { Given the variatiol } \\
\text { in countries' } \\
\text { capacity to respor } \\
\text { to infectious } \\
\text { diseases, public } \\
\text { health measures } \\
\text { must be adapted } 1 \\
\text { the context of any } \\
\text { given country. }\end{array}$ \\
\hline
\end{tabular}




\begin{tabular}{|c|c|c|c|c|c|c|c|c|}
\hline Author/ Date & Title & Joumal & $\begin{array}{l}\text { Type of } \\
\text { study/report }\end{array}$ & $\begin{array}{l}\text { Peer } \\
\text { review } \\
\text { status }\end{array}$ & Topic & Setting & $\begin{array}{l}\text { Outcome of } \\
\text { interest }\end{array}$ & Main message \\
\hline $\begin{array}{l}\text { Shakespeare } \\
\text { et al, August } \\
2020\end{array}$ & $\begin{array}{l}\text { Resilience } \\
\text { and } \\
\text { vulnerability } \\
\text { of maternity } \\
\text { services in } \\
\text { Zimbabwe: a } \\
\text { comparative } \\
\text { analysis of } \\
\text { the effect of } \\
\text { COVID-19 } \\
\text { and } \\
\text { lockdown } \\
\text { control } \\
\text { measures on } \\
\text { maternal and } \\
\text { perinatal } \\
\text { outcomes at } \\
\text { Mpilo Central } \\
\text { Hospital. }\end{array}$ & $\begin{array}{l}\text { Research } \\
\text { Square } \\
\text { preprint }\end{array}$ & $\begin{array}{l}\text { Retrospective } \\
\text { study }\end{array}$ & Preprint & $\begin{array}{l}\text { Effects on } \\
\text { pregnancy, } \\
\text { labour and } \\
\text { delivery }\end{array}$ & Zimbabwe & $\begin{array}{l}\text { Maternal and } \\
\text { perinatal } \\
\text { morbidity and } \\
\text { mortality }\end{array}$ & $\begin{array}{l}\text { Lockdown } \\
\text { measures did not } \\
\text { increase maternal } \\
\text { severe maternal } \\
\text { morbidity. There } \\
\text { was a minor } \\
\text { increase in early } \\
\text { neonatal death. } \\
\text { There is a need fo } \\
\text { continuous } \\
\text { monitoring of thes } \\
\text { trends. }\end{array}$ \\
\hline $\begin{array}{l}\text { Ezenwa et al., } \\
\text { May } 2020\end{array}$ & $\begin{array}{l}\text { Management } \\
\text { of COVID-19: } \\
\text { a practical } \\
\text { guideline for } \\
\text { maternal and } \\
\text { newborn } \\
\text { health care } \\
\text { providers in } \\
\text { Sub-Saharan } \\
\text { Africa }\end{array}$ & $\begin{array}{l}\text { The journal } \\
\text { of maternal- } \\
\text { fetal \& } \\
\text { neonatal } \\
\text { medicine }\end{array}$ & $\begin{array}{l}\text { Clinical } \\
\text { guidelines }\end{array}$ & $\begin{array}{l}\text { Peer } \\
\text { reviewed }\end{array}$ & $\begin{array}{l}\text { Direct effects } \\
\text { on pregnancy, } \\
\text { labour and } \\
\text { delivery }\end{array}$ & Nigeria & $\begin{array}{l}\text { Maternal and } \\
\text { newborn clinical } \\
\text { guidelines. }\end{array}$ & $\begin{array}{l}\text { A Clinical guidelin } \\
\text { (aligned with WHC } \\
\text { COVID-19 } \\
\text { management } \\
\text { objectives) has be } \\
\text { proposed. The } \\
\text { guideline will be } \\
\text { revised as new } \\
\text { evidence and } \\
\text { information about } \\
\text { COVID-19 in } \\
\text { maternal and } \\
\text { newborn care } \\
\text { emerges }\end{array}$ \\
\hline
\end{tabular}

\section{Themes}

We identified 3 major themes: delayed care, disruption in service provision and utilization and mitigation strategies/recommendations.

\section{Delayed care}

Most studies reported delayed maternal and child health care as a major impact of the COVID-19 pandemic. Oluwasola and colleagues reported that reproductive, maternal, newborn and child health service provision and utilization have decreased substantially, resulting in delayed necessary care. The authors explain that in a pandemic, pregnant women are faced with the uncertainty to attend a clinic (25). Murewanhema and colleagues also provided a conceptualization of delay across the cascade of maternity care from antenatal care to postnatal care (26). Reporting on the experience of a large hospital in Malawi, Nadi and colleagues explained that elective operations, referrals and clinic visits have been minimized, in addition to delays in treatment follow-up and drug supply (27).

\section{Disruption in service provision and utilization}

The Disruption in service provision and utilization was a major theme illuminated in many of the included studies. Shikuku and colleagues reported reduced utilization of long-term contraceptives like implants and intrauterine devices in Kenya (28). Ogunkola and colleagues highlighted the closure of 5,633 static and mobile health clinics and community-based care centers of the International Planned Parenthood Federation across 64 countries due to the COVID-19 pandemic (29).

Nadi and colleagues (27) also reported a 75 percent reduction in paediatric bed occupancy and a loss in national and international medical staff in a large medical institution in Malawi. Pires and colleagues also reported reductions in maternity hospital deliveries, childhood vaccination, family planning visit, antenatal visit and increases in home deliveries in Nampula, Mozambique (30).

In a retrospective study comparing routine monthly maternal and perinatal statistics three months before and after COVID-19 emergency measures at a government tertiary referral hospital in Zimbabwe, Shakespeare and colleagues report that between January-March, and April-June 2020, average monthly deliveries fell from 747 to 681 and Caesarean section rates from 29.8-26.6\% (31). Banke-Thomas and colleagues reported that COVID-19 has directly impacted cost of maternity service utilization; costs of spontaneous vaginal delivery and cesarean delivery have doubled and tripled, respectively during the pandemic, compared to pre-COVID-19 (32).

Fear of COVID-19 has also been reported as predictors of antenatal care service utilization (33). Oluwasola and Bello's review also highlights the potential negative implications of COVID-19 for obstetric and gynecologic practice in Africa (25).

\section{Mitigation strategies and recommendations}

Although only one of the included studies was a guidance document (protocol), most of the included studies offered strategies and measures to minimize the impact of the COVID-19 pandemic on maternal and child health. Murewanhema and colleagues suggested that a formal assessment is needed to determine 
the true impact of COVID-19 on maternity outcomes, warning that mitigation strategies should be expedited to avert further damage (26).

Telemedicine and other e-health tools were suggested as a feasible alternative for triaging obstetric patients in a pandemic. Murewanhema and colleagues suggested internet subsidies for maternity care to optimize use since financial barriers may prevent this utilization (26). Despite the potential benefits of telemedicine, Pallangyo et al. reported that telehealth and phone triage for antenatal clinics are non-existent in rural areas (34).

Highlighting the gendered nature of the impact of COVID-19, the role of African civil society organizations in protecting sexual and reproductive health was underscored by Eghtessadi and colleagues (35). They proposed five action principles including: 1) accountability of governments around optimal use of COVID-19 funds and resources; 2) keeping sexual and reproductive health and HIV central to the Universal Health Coverage agenda; 3 ) lobbying governments to invest in innovative and good practice models; 4) mitigate the gendered impact of COVID-19 and 5) advocate that African governments remain vigilant in securing access to new therapeutics at reasonable cost from the global market.

\section{Discussion}

As the COVID-19 pandemic continues to stretch the health care systems of many countries, we note a need to be mindful of its direct and indirect effects on maternal and child health in Africa, given the already fragile health care systems across the continent. Africa constitutes about $11 \%$ of the world's population but has $60 \%$ and $90 \%$ of world's population living with HIV/AIDS and Malaria respectively. The continent also contributes the most to the global burden of maternal and child morbidity, as well as mortality (36). The overall ramification of the pandemic in this region, therefore, may be more protracted than other regions. These poor outcomes reflect the impact of poor health systems financing, governance, and information systems, as well as the poor access to medical products, vaccines and technology and service delivery.

Our scoping review shows that not much has been written or published on this topic. As many health systems deal with the ramification of COVID-19, strategies have been adopted to limit its devastating effects. Wangamati and Sandby report how some of these measures are being adopted in two counties in Kenya to enable transportation of pregnant women in labour to hospitals even during a curfew (37).

Available publications included in this review indicate that time-sensitive programs such as maternity services, immunization and reproductive health services that are essential to the wellbeing of mothers and children have not been prioritized during the pandemic. Although restrictions and other mitigation measures were expected to reduce the spread of the coronavirus, it resulted in poor access to care and low utilization of maternal and child health services, including reproductive services. For example, in Kenya, it was recorded that there was high utilization of short-term family planning services such as injectables and pills, and in the long-term, there was a rise in the cases of caesarean Sect. (28). As recorded by Pallangyo and colleagues, rural areas were worst hit by the strict stay-at-home measures put in places such as Kenya, Uganda and Tanzania. Strategies like phone and telehealth option which provide counselling services for new mothers and information for people with poor obstetric history and emergency medical problems were inaccessible in rural areas, leading to late presentation of pregnant women to hospital, more cases of stillbirths and preterm birth. In addition, reports of inadequate training for midwives on COVID19 protocol and standard operational guidelines on $\mathrm{MNCH}$, further worsened the situation at this level of service delivery (34).

The disproportionate impact of the pandemic on the health of the most vulnerable population segments is consistent with global reports which suggests that pregnant women, infants, children, and the elderly are more likely to be affected by infectious diseases than other people, especially in low-income countries. Moreover, a WHO report on acute respiratory infections suggests that infections with pandemic potential requires prompt changes to treatment center plans to include, triage, repurposing, capacity building and awareness creation for personnel and clients to reduce public health risks and health disparity associated with the disease (38). In the southern African region, on the flipside, there was a report of increased use of home-based deliveries, which calls into question what strategies were most suited to safeguard the continued delivery of routine health programs while preventing the adverse effect of the pandemic on maternal and child health.

We observed that only one publication (39), focused on aligning country health programs with WHO COVID-19 management guidelines. The recommendations include providing countries with updated information on emerging scientific knowledge to help decision-makers implement prompt changes to health systems based on country context. Despite the realigned guidelines, there were problems associated with increased cost of antenatal and maternal care leading to disproportionate access to public health services. Other challenges mentioned were the reduction in international donations and low uptake of prenatal and maternal services.

The sum of these issues highlighted in this research shows that there are opportunities to be derived if civil society organizations are engaged in monitoring adherence to recommended guidelines to play a role in keeping governments accountable (35). Also, the introduction of telemedicine helped to cushion the impact of COVID-19 on wellbeing.

There are, however, gaps in the operationalization of $\mathrm{MNCH}$ programs and services across the region as shown in the studies reviewed. Lack of emergency preparedness plans for human and material resources tailored to peculiar health system gaps, and health needs remain an ongoing challenge in African regions. This weakness will need to be urgently addressed to reduce additional maternal, neonatal, and child health risks during global health emergencies.

The comprehensive and rigorous nature of our search strategy and scoping review methodology are major strengths of this review. The duplicate screening by two authors with $\mathrm{MNCH}$ expertise and review experience is another strength. With respect to study limitations, potential delay in indexing might have excluded some relevant studies. We did not appraise the quality of the methodology of each study since our priority was to rapidly compile evidence of this evolving topic and our strategy was limited to English papers published between January 2020 and February 2021.

\section{Conclusion}


This scoping review sought to document the effects COVID-19 pandemic had on maternal and child health in relation to health care access, utilization, and health outcome in Africa. This review identified limited research on this topic, however, the findings suggest that despite the various mitigation strategies and recommendations adopted across jurisdictions, the impact on health outcomes and health systems is cause for concern. The inflated cost of maternal and prenatal care, midwifery practice and neonatal care, including poor access to sexual and reproductive health care, are few indirect and direct effects on outcomes. The implication includes continuous disruptions in services and delayed or lack of access to needed care for women and children. Our review has shown that maternity services, immunization, and reproductive health services that promote the wellbeing of mothers and children have not been prioritized during the pandemic. There is a continuing need for health systems capacity expansion - with built-in redundancies - to shield the continent's most vulnerable population segments from the shocks of the current COVID-19 pandemic and future global health emergencies.

\section{Abbreviations}

COVID-19: Coronavirus disease 2019

MNCH: maternal neonatal and child health

SARS-CoV-2: severe acute respiratory syndrome coronavirus 2

WHO: World Health Organization

\section{Declarations}

\section{Authors' Contributions}

PA and AO conceptualized and designed the study. LS led the literature searches with support from AO. Literature search, data screening and data extraction were verified by PA, LS, TA, SA and AO. Results and summary of the studies were done by PA and SA. AO wrote the introduction section of the manuscript, while LS wrote its methodology. The results section was written by PA and SA, while the discussion was written by PA, LS, SA and TA. All authors revised the manuscript critically for cogent intellectual content and manuscript preparation prior to submission.

\section{Ethics Approval and Consent to Participate.}

Not applicable in review.

\section{Consent for Publication}

Not applicable in review.

\section{Availability of Data and materials.}

Full review will be made available.

\section{Competing interests}

The authors declare that they have no competing interests.

\section{Funding}

N/A.

\section{References}

1. World Health Organization. WHO Director-General's opening remarks at the media briefing on COVID-19-11 March 2020 [Internet]. 2020 [cited 2021 May 25]. Available from: https://www.who.int/director-general/speeches/detail/who-director-general-s-opening-remarks-at-the-media-briefing-on-covid-19--11march-2020.

2. Deb P, Nadeem S, Peiris JS. The Effect of Containment Measures on the COVID-19 Pandemic [Internet]. 2021 Jan [cited 2021 May 25]. Available from: https://www.imf.org/en/Publications/WP/Issues/2020/08/07/The-Effect-of-Containment-Measures-on-the-COVID-19-Pandemic-49572.

3. International Monetary Fund. Policy Responses to COVID19 [Internet]. IMF. 2021 [cited 2021 May 25]. Available from: https://www.imf.org/en/Topics/imfand-covid19/Policy-Responses-to-COVID-19.

4. World Health Organization. Maintaining essential health services: operational guidance for the COVID-19 context interim guidance [Internet]. 2020 [cited 2021 May 25]. Available from: https://www.who.int/publications-detail-redirect/WHO-2019-nCoV-essential-health-services-2020.1.

5. World Health Organization. Pulse survey on continuity of essential health services during the COVID-19 pandemic: interim report, 27 August 2020 [Internet]. 2020 [cited 2021 May 25]. Available from: https://www.who.int/publications-detail-redirect/WHO-2019-nCoV-EHS_continuity-survey-2020.1.

6. Ncayiyana DJ. Doctors and nurses with HIV and AIDS in sub-Saharan Africa. BMJ. 2004 Sep;9(7466):584-5. 329(.

7. O'Hara LM, Yassi A, Zungu M, Malotle M, Bryce EA, Barker SJ, et al. The neglected burden of tuberculosis disease among health workers: a decade-long cohort study in South Africa. BMC Infect Dis. 2017 Aug 7;17(1):547. 
8. Elston JWT, Cartwright C, Ndumbi P, Wright J. The health impact of the 2014-15 Ebola outbreak. Public Health. 2017 Feb;143:60-70.

9. Ifeagwu SC, Yang JC, Parkes-Ratanshi R, Brayne C. Health financing for universal health coverage in Sub-Saharan Africa: a systematic review. Glob Health Res Policy. 2021 Mar 1;6(1):8.

10. Sambo LG, Kirigia JM, Ki-Zerbo G. Health financing in Africa: overview of a dialogue among high level policy makers. BMC Proc. 2011 Jun 13;5 Suppl 5:S2.

11. United Nations Statistics Division. The Sustainable Development Goals Report 2020 [Internet]. [cited 2021 May 25]. Available from: https://unstats.un.org/sdgs/report/2020/goal-03/.

12. Alkema L, Chou D, Hogan D, Zhang S, Moller A-B, Gemmill A, et al. Global, regional, and national levels and trends in maternal mortality between 1990 and 2015, with scenario-based projections to 2030: a systematic analysis by the UN Maternal Mortality Estimation Inter-Agency Group. Lancet Lond Engl. 2016 Jan 30;387(10017):462-74.

13. United Nations Children's Fund. Levels and trends in child mortality 2020 [Internet]. 2020 [cited 2021 May 25]. Available from: https://www.unicef.org/reports/levels-and-trends-child-mortality-report-2020.

14. Ahmed SAKS, Ajisola M, Azeem K, Bakibinga P, Chen Y-F, Choudhury NN, et al. Impact of the societal response to COVID-19 on access to healthcare for non-COVID-19 health issues in slum communities of Bangladesh, Kenya, Nigeria and Pakistan: results of pre-COVID and COVID-19 lockdown stakeholder engagements. BMJ Glob Health. 2020 Aug;5(8).

15. Graham WJ, Afolabi B, Benova L, Campbell OMR, Filippi V, Nakimuli A, et al. Protecting hard-won gains for mothers and newborns in low-income and middle-income countries in the face of COVID-19: call for a service safety net. BMJ Glob Health [Internet]. 2020 Jun 3 [cited 2021 May 25];5(6). Available from: https://www.ncbi.nlm.nih.gov/pmc/articles/PMC7298807/.

16. Kotlar B, Gerson E, Petrillo S, Langer A, Tiemeier H. The impact of the COVID-19 pandemic on maternal and perinatal health: a scoping review. Reprod Health [Internet]. 2021 Jan 18 [cited 2021 May 16];18. Available from: https://www.ncbi.nlm.nih.gov/pmc/articles/PMC7812564/.

17. Erdem H, Lucey DR. Healthcare worker infections and deaths due to COVID-19: A survey from 37 nations and a call for WHO to post national data on their website. Int J Infect Dis. 2021 Jan 1;102:239-41.

18. Menendez C, Gonzalez R, Donnay F, Leke RGF. Avoiding indirect effects of COVID-19 on maternal and child health. Lancet Glob Health. 2020 Jul 1;8(7):e863-4.

19. UNESCO. Adverse consequences of school closures [Internet]. UNESCO. 2020 [cited 2021 May 25]. Available from: https://en.unesco.org/covid19/educationresponse/consequences.

20. Figueroa JP, Bottazzi ME, Hotez P, Batista C, Ergonul O, Gilbert S, et al. Urgent needs of low-income and middle-income countries for COVID-19 vaccines and therapeutics. The Lancet. 2021 Feb;13(10274):562-4. 397(.

21. Kupferschmidt K. 2021, Pm 2:45. Unprotected African health workers die as rich countries buy up COVID-19 vaccines [Internet]. Science | AAAS. 2021 [cited 2021 May 25]. Available from: https://www.sciencemag.org/news/2021/02/unprotected-african-health-workers-die-rich-countries-buy-covid-19vaccines.

22. Akseer N, Kandru G, Keats EC, Bhutta ZA. COVID-19 pandemic and mitigation strategies: implications for maternal and child health and nutrition. Am J Clin Nutr. 2020 Aug;112(2)(1):251-6.

23. Roberton T, Carter ED, Chou VB, Stegmuller AR, Jackson BD, Tam Y, et al. Early estimates of the indirect effects of the COVID-19 pandemic on maternal and child mortality in low-income and middle-income countries: a modelling study. Lancet Glob Health. 2020 Jul 1;8(7):e901-8.

24. Arksey H, O'Malley L. Scoping studies: towards a methodological framework. Int J Soc Res Methodol. 2005 Feb;1(1):19-32. 8(.

25. Oluwasola TAO, Bello 00. COVID-19 and its implications for obstetrics and gynecology practice in Africa. Pan Afr Med J [Internet]. 2021 Jan 7 [cited 2021 May 16];38(15). Available from: https://www.panafrican-med-journal.com/content/article/38/15/full.

26. Murewanhema G, Nyakanda MI, Madziyire MG. Restoring and maintaining robust maternity services in the COVID-19 era: a public health dilemma in Zimbabwe. Pan Afr Med J [Internet]. 2020 Nov 8 [cited 2021 May 16];37(Suppl 1). Available from:

https://www.ncbi.nIm.nih.gov/pmc/articles/PMC7796837/.

27. Nandi B, Schultz A, Huibers MH, Msekandiana A, Chiume-Kayuni M. SARS-CoV-2 in Malawi: Are we sacrificing the Youth in sub-Saharan Africa? J Glob Health. 2020 Dec;10(2):020336.

28. Shikuku D, Nyaoke I, Gichuru S, Maina O, Eyinda M, Godia P, et al. Early indirect impact of COVID-19 pandemic on utilization and outcomes of reproductive, maternal, newborn, child and adolescent health services in Kenya. medRxiv. 2020 Sep 9;2020.09.09.20191247.

29. Ogunkola IO, Adebisi YA, Imo UF, Odey GO, Esu E, Lucero-Prisno DE. Impact of COVID-19 pandemic on antenatal healthcare services in Sub-Saharan Africa. Public Health Pract. 2021 Nov;1:2:100076.

30. Pires P, Macaringue C, Ahmed A, Mucufo J, Mupueleque M, Siemens R, et al. Covid-19 Pandemic Impact on Maternal and Child Health Services Access in Nampula. Mozambique: A Mixed Methods Research; 2020.

31. Shakespeare C, Dube H, Moyo S, Ngwenya S. Resilience and vulnerability of maternity services in Zimbabwe: a comparative analysis of the effect of Covid-19 and lockdown control measures on maternal and perinatal outcomes at Mpilo Central Hospital. [Internet]. 2020 [cited 2021 May 25]. Available from: https://www.researchsquare.com.

32. Banke-Thomas A, Makwe CC, Balogun M, Afolabi BB, Alex-Nwangwu TA, Ameh CA. Utilization cost of maternity services for childbirth among pregnant women with coronavirus disease 2019 in Nigeria's epicenter. Int J Gynaecol Obstet Off Organ Int Fed Gynaecol Obstet. 2021 Feb;152(2):242-8.

33. Tadesse E. Antenatal Care Service Utilization of Pregnant Women Attending Antenatal Care in Public Hospitals During the COVID-19 Pandemic Period. Int J Womens Health. 2020 Dec 8;12:1181-8. 
34. Pallangyo E, Nakate MG, Maina R, Fleming V. The impact of covid-19 on midwives' practice in Kenya, Uganda and Tanzania: A reflective account. Midwifery. 2020 Oct;89:102775.

35. Eghtessadi R, Mukandavire Z, Mutenherwa F, Cuadros D, Musuka G. Safeguarding gains in the sexual and reproductive health and AIDS response amidst COVID-19: The role of African civil society. Int J Infect Dis IJID Off Publ Int Soc Infect Dis. 2020 Nov;100:286-91.

36. World Health Organization. WHO I The African Regional Health Report: The Health of the People [Internet]. WHO. World Health Organization; 2021 [cited 2021 May 31]. Available from: http://www.who.int/bulletin/africanhealth/en/.

37. Wangamati CK, Sundby J. The ramifications of COVID-19 on maternal health in Kenya. Sex Reprod Health Matters. 2020 Jan 1;28(1):1804716.

38. World Health Organization. Severe Acute Respiratory Infections Treatment Centre [Internet]. 2020 Mar [cited 2021 May 25]. Available from: https://www.who.int/publications-detail-redirect/10665-331603.

39. Ezenwa BN, Fajolu IB, Akinajo OR, Makwe CC, Oluwole AA, Akase IE, et al. Management of covid-19: a practical guideline for maternal and newborn health care providers in Sub-Saharan Africa. J Matern Fetal Neonatal Med. 2020 May;18(0):1-7. 0(.

\section{Figures}

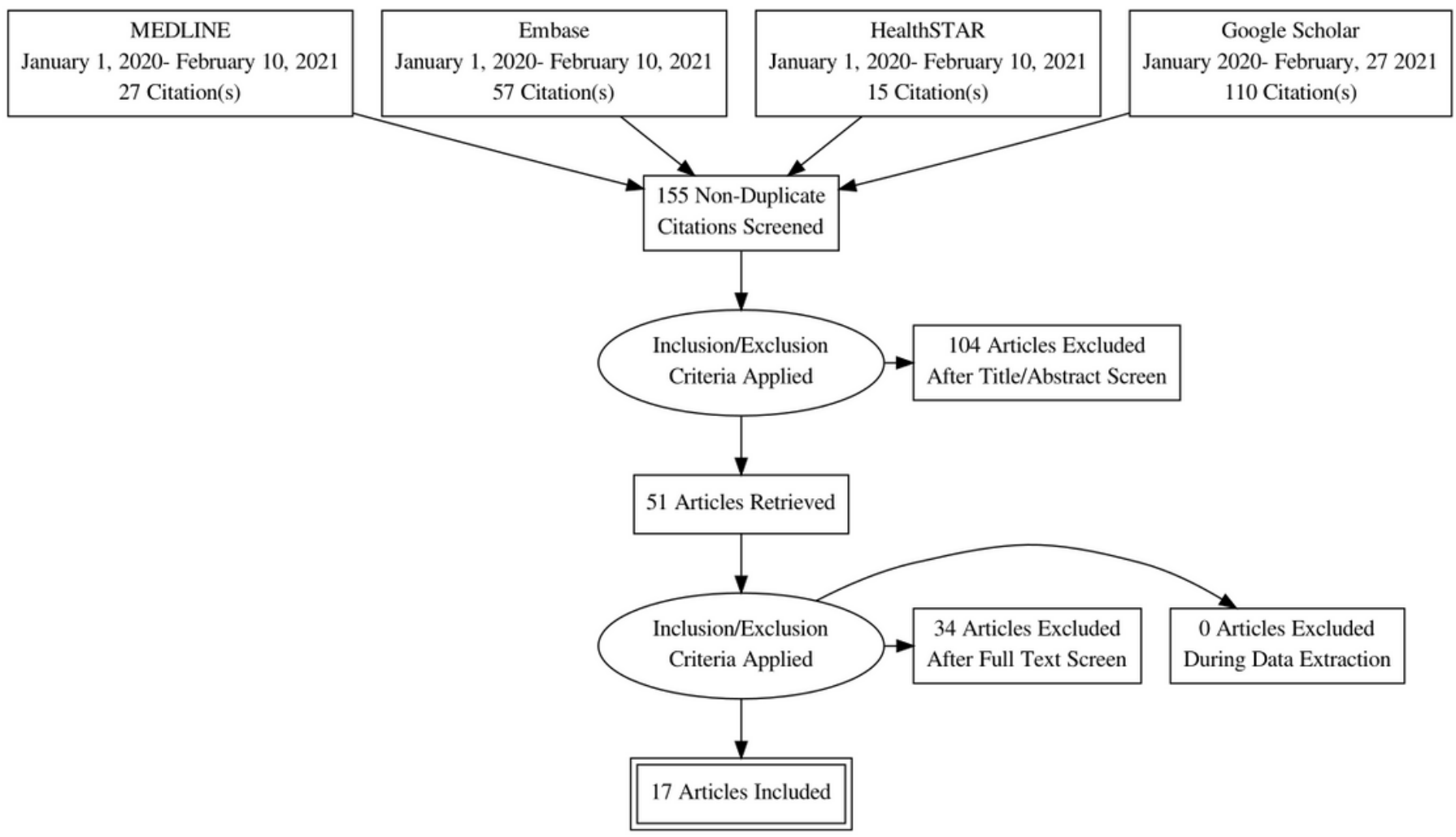

Figure 1

Flowchart of literature selection

\section{Supplementary Files}

This is a list of supplementary files associated with this preprint. Click to download.

- Additonalfile1SearchStrategy.docx 